\title{
APTE: identification of indirect read-out A-DNA promoter elements in genomes
}

\author{
David C Whitley², Valeria Runfola', Peter Cary ${ }^{1}$, Liliya Nazlamova', Matt Guille ${ }^{1}$ and Garry Scarlett ${ }^{1 *}$
}

\begin{abstract}
Background: Transcriptional regulation is normally based on the recognition by a transcription factor of a defined base sequence in a process of direct read-out. However, the nucleic acid secondary and tertiary structure can also act as a recognition site for the transcription factor in a process known as indirect read-out, although this is much less understood. We have previously identified such a transcriptional control mechanism in early Xenopus development where the interaction of the transcription factor ilf3 and the gata2 promoter requires the presence of both an unusual A-form DNA structure and a CCAAT sequence. Rapid identification of such promoters elsewhere in the Xenopus and other genomes would provide insight into a less studied area of gene regulation, although currently there are few tools to analyse genomes in such ways.

Results: In this paper we report the implementation of a novel bioinformatics approach that has identified 86 such putative promoters in the Xenopus genome. We have shown that five of these sites are A-form in solution, bind to transcription factors and fully validated one of these newly identified promoters as interacting with the ilf3 containing complex CBTF. This interaction regulates the transcription of a previously uncharacterised downstream gene that is active in early development.

Conclusions: A Perl program (APTE) has located a number of potential A-form DNA promotor elements in the Xenopus genome, five of these putative targets have been experimentally validated as A-form and as targets for specific DNA binding proteins; one has also been shown to interact with the A-form binding transcription factor ilf3. APTE is available from http://www.port.ac.uk/research/cmd/software/ under the terms of the GNU General Public License.
\end{abstract}

Keywords: A-form DNA, Transcription, Xenopus, Promoter analysis

\section{Background}

Transcription is the major level at which cellular protein concentration is regulated in response to environmental and developmental cues. Transcriptional control is mediated by the interaction of transcription factors and DNA elements. These elements are normally one instance of a set of similar sequences (or motifs) that the transcription factor 'reads' in a process known as direct read-out. There are some cases, however, where the transcription factor recognises not the sequence per se but the structure that the DNA adopts as a consequence of both sequence and conditions. The disruption of the DNA from the standard B-form conformation acts as a recognition

\footnotetext{
* Correspondence: garry.scarlett@port.ac.uk

'Biophysics Laboratories, School of Biological Sciences, Institute of Biomedical and Biomolecular Science, University of Portsmouth, King Henry Building, King Henry I Street, Portsmouth PO1 2DY, UK

Full list of author information is available at the end of the article
}

site for the transcription factor in a process known as indirect read-out. This is well established in prokaryotes [1-3] but less recognised in eukaryotic cells, although an indirect read-out mechanism has been suggested for a selection of eukaryotic gene promoters [4-6]. Given the size of vertebrate genomes it is highly likely that some regions consist of sequences forming non-canonical structures and that some of these are regulatory. Indeed local DNA topography has been shown to correlate better than sequence with functional non-coding regions of the human genome [7].

The canonical double-stranded DNA structure is B-form, a right-handed helix with 3.4 A between base pairs and a base tilt of 6 degrees to the helix axis. However, DNA can exist in a number of other conformations, the major types being A-form, Z-form and tetraplex, all of which have been implicated in gene regulation [8-10]. A-form is the canonical dsRNA structure with 
right-handed helices but with only $2.6 \AA$ between bases and a 20-degree base tilt, while the sugar in A-form is in the $\mathrm{c}-3^{\prime}$ endo position in contrast to the $\mathrm{c}-2^{\prime}$ endo position observed for B-form. These differences lead to A-form helices being 'shorter and fatter', possessing major and minor grooves of similar width and the major groove deepened with respect to the B-form structure. Although DNA is usually in the canonical B-form it can be induced into A-form by dehydration and certain DNA sequences can naturally adopt an A-form helix under physiological conditions [11]. These A-form elements can then be specifically recognised by DNA binding proteins.

The interaction of the Xenopus CCAAT box transcription factor (CBTF) complex and the promoter of the developmentally important gata 2 gene is an example of a transcriptional regulatory mechanism involving A-form DNA. We have previously shown that this mechanism is based on an interaction requiring both DNA base specific (direct read-out) and DNA structure specific (indirect read-out) interactions $[8,6]$. The CBTF complex is composed of approximately eight sub-units of which the ilf3 protein is currently the only published component; however, this subunit is critical for CBTF activity. Ilf3 is found in the nucleus when the gata2 gene, a developmentally regulated gene involved in blood formation, is transcribed. A number of biochemical experiments have also confirmed ilf3 as a regulator of gata2 transcription, including chromatin associated precipitation (ChIP) identifying ilf3 at the gata2 promoter during active transcription of this gene [12]. Therefore the CBTF complex and its interactions is of interest both from developmental and transcriptionally mechanistic viewpoints.

Ilf3 contains two double stranded RNA binding domains (dsRBDs) and these domains are required for transcriptional activation in vivo and DNA binding in vitro [8]. The RNA binding activity of ilf3, and other dsRBD containing proteins, has been well characterised, indeed ilf3 was first identified through its interaction with RNA [13]. Crystal and NMR structures of a dsRBD alone exist [14], as does a crystal structure of the protein-RNA complex [15]. Alongside saturation mutagenesis studies, these structural studies have revealed that the domains recognise the A-form helical structure of double stranded RNA, although far less is known about their interaction with DNA. We have previously shown that Xenopus ilf3 contributes to the activity of CBTF as a transcriptional activator by its interaction with structure-specific DNA sequences. Specifically the dsRBDs of ilf3 are capable of interacting not only with A-form RNA but also non-canonical A-form DNA, such as that at the gata2 promoter [6].

Here we report the development and validation of a bioinformatics tool for the analysis of genomic data to identify other potential promoters that utilise an A-form
DNA structural component; in particular, those that are responsive to the transcription factor ilf3.

\section{Results and discussion}

\section{Predicted promoter elements}

We implemented our search program based on the A-form prediction algorithm of Basham et. al [11] but including our previously described modifications [8]. This program was used to search the Xenopus tropicalis JGI 4.2 genome assembly for putative A-form promoters. Searches were further restricted to a $500 \mathrm{bp} 5^{\prime}$ proximity of a start site of a transcribed unit and also to a variety of motifs known to be common transcription factor binding sequences. The prediction of A-form DNA is based on the $A-D N A$ propensity energy (APE), a numerical measure derived from solvent free energy calculations that indicates the thermodynamic propensity for a sequence to adopt the A-DNA conformation. The APE value at position $i$ in a DNA sequence depends on the central base $b_{i}$ and the $5^{\prime}\left(b_{i-1}\right)$ and 3' $\left(b_{i+1}\right)$ flanking bases. From a triplet code of APE values for tri-nucleotides, the APE value for each base-pair is calculated (in $\mathrm{kcal} / \mathrm{mol}$ ) as the sum of the triplet APE values for the forward and reverse strands. In our process we have defined the predicted A-form promoter sequence (APS) as a sequence with consecutive negative APE values, together with the two flanking bases required for the APE calculation. Given a direct read-out promoter motif, for each gene the algorithm searches a region upstream of the transcription start site (TSS) for instances of the motif or its reverse complement preceded by an APS of pre-specified minimum length, with the APS and motif separated by at most a pre-specified maximum distance. The combined promoter sequence (CPS) extends from the start of the APS to the end of the motif (Figure 1).

We selected APS sequences of length $\geq 12$ bp preceding several common promoter sequence motifs by at most 20 positions and within $500 \mathrm{bp}$ of a TSS. A minimum APS of $12 \mathrm{bp}$ was selected as our preliminary experimental

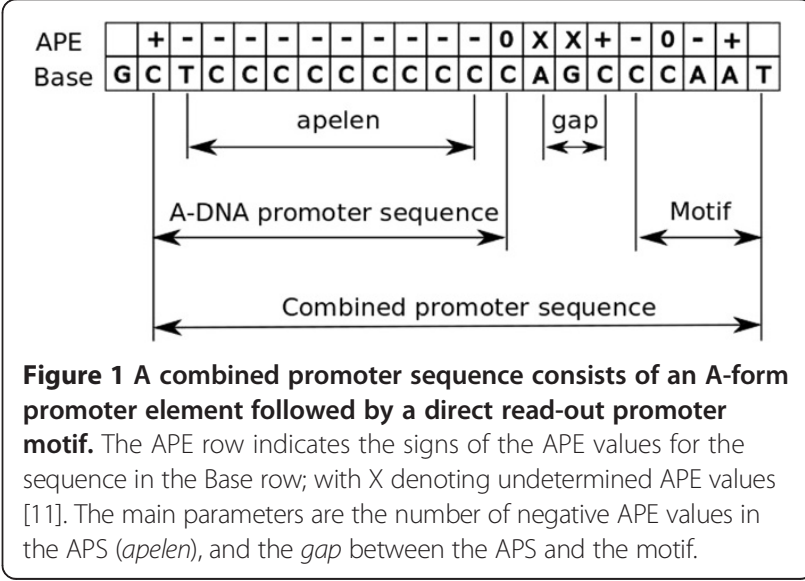


studies show that this length of APS reliably gives an A-form structure as identified by circular dichroism (manuscript in preparation), while a limit of $20 \mathrm{bp}$ between the APS and motif is based on the known footprint of the CBTF complex [8]. The number of APS and CPS (for the motifs CCAAT, GGGCGG, AGATA and TGATA) in the 4.2 assembly of the Xenopus tropicalis genome are shown in Table 1 along with their frequencies in regions 500 bp upstream of a TSS. The frequencies of the four motifs, in the whole genome and constrained to CPS or regions $500 \mathrm{bp}$ upstream of a TSS, are shown in Table 2, the full list of hits is provided in Table 3. In general the CCAAT, AGATA and TGATA motifs occur with high frequency and in many cases several instances of a motif are found preceding a gene. The A-DNA promoter sequences, however, occur before only $3.2 \%$ of genes. An APS therefore occur only rarely in comparison with the motifs, and the combined promoter sequences only appear before approximately $0.47 \%$ of genes. Monte Carlo simulation of $10^{6}$ sequences of $500 \mathrm{bp}$ selected randomly according to the nucleotide frequencies in the Xenopus tropicalis genome (0.299733 (A), 0.200318 (C), 0.200317 (G) and 0.299632(T)) produced expected numbers of 5.90 APS and 1.49 CPS in the genome. Thus we estimate that there are almost 100 times more APS and over 50 times more CPS in regions 500 bp upstream of TSS in the Xenopus tropicalis genome than would be expected by chance.

\section{Selection and validation of a predicted promoter}

Having identified 86 putative promoter elements in the JGI 4.2 assembly we randomly selected five for validation. The 36 bp sequences corresponding to the five selected CPSs are shown in Figure 2 with their predicted transcription factor binding sites. Experimentally we confirmed that these sequences were (i) A-form in character and (ii) indeed a target for a DNA binding protein.

Circular Dichroism experimental studies of all five selected sequences confirm that these GC-rich duplexes are largely in the A-form conformation. The data shows two strong positive bands with maxima between 186$189 \mathrm{~nm}$ and 267-269 $\mathrm{nm}$ respectively for all five constructs

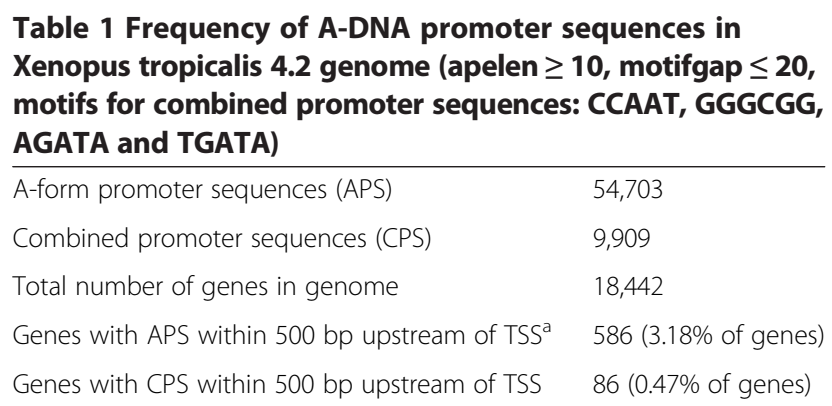

${ }^{\mathrm{a}}$ Transcription Start Site.
Table 2 Frequency of motifs in combined promoter sequences (CPS) in Xenopus tropicalis 4.2 genome (apelen $\geq 10$, motifgap $\leq 20$ )

\begin{tabular}{lllll}
\hline Motif & CCAAT & GGGCGG & AGATA & TGATA \\
\hline $\begin{array}{l}\text { Genes with motif within } \\
\text { 500 bp upstream of TSS }\end{array}$ & 13,255 & 2,531 & 12,703 & 12,201 \\
$\begin{array}{l}\text { Total number of motifs } \\
\text { in genome }\end{array}$ & $1,814,253$ & 108,168 & $1,918,291$ & $1,617,806$ \\
$\begin{array}{l}\text { Motifs within } 500 \mathrm{bp} \\
\text { upstream of TSS }\end{array}$ & $\mathbf{2 5 , 2 5 3}$ & 3,377 & 23,471 & 20,927 \\
(including multiples) & $(1.39 \%)$ & $(3.12 \%)$ & $(1.22 \%)$ & $(1.29 \%)$ \\
Motifs in CPS & 3,771 & 1,080 & 2,351 & 2,707 \\
& $(0.21 \%)$ & $(1.00 \%)$ & $(0.12 \%)$ & $(0.17 \%)$ \\
$\begin{array}{l}\text { Motifs in CPS within } \\
\text { 500 bp upstream of TSS }\end{array}$ & $(0.002 \%)$ & $(0.012 \%)$ & $(0.001 \%)$ & $(0.001 \%)$ \\
\hline
\end{tabular}

${ }^{\mathrm{a}}$ Transcription Start Site.

with a negative band minima between $240-243 \mathrm{~nm}$, these spectra are indicative of A-form. The absence of a clear, strong positive band at $180-186 \mathrm{~nm}$ suggest there is little B-form DNA duplex present in any of the five sequences, although there is weak positive contribution between 180$190 \mathrm{~nm}$ for thrsp, obp, kif27 and gdi3 causing a slight distortion to the main positive band (260 nm to $300 \mathrm{~nm}$ ). Further, the intensity of the band maxima at (267-269 nm) is significantly more positive than expected for B-form $(+2.5$ to 3.3$)$ and the experimental ellipticity values are more typical of A-form duplexes (+4.3 to 6.86). Using the triple base APE prediction for A and B-form DNA duplexes all five selected DNA sequences have strong continuous A-form runs upstream of the CCAAT, AGATA and GGGCGG motifs. These continuous A-form regions only represent 28 to $39 \%$ of the total duplexes in the A-form for all five sequences, the $C D$ measurements suggest that the A-form content is at least between 50 to $80 \%$ for all five duplexes. Using the triple base APE prediction for A and B-form Dna duplexes the total A-form prediction content for Gtf2e1.2 for example is $56 \%$ with $20 \%$ having no bias for A or B-form, $14 \%$ undetermined APE values, $11 \%$ with a preference for B-form duplexes. This would suggest the minimum A-form content is $56 \%$ and may be as high as $85 \%$, however in all cases the duplexes are mainly in the A-form conformation.

We next tested that these oligonucleotides were specific targets for DNA binding proteins such as transcription factors. Radiolabelled sequences were mixed with whole embryo extract and electrophoretic mobility shift (EMSA) assays were performed. All the sequences found specific complexes with embryo extract, these complexes were competed by unlabelled self-competitor. However they were not competed by an alternative 36 bp competitor that contained a CCAAT box sequence but which was strongly B-form in structure (Figure $3 \mathrm{a}$ and $\mathrm{b}$ ). Having shown that all five of the selected sequences were both A-form and targets for specific DNA binding proteins 
Table 3 Gene IDs and names of the immediately downstream genes of the $\mathbf{8 6}$ putative A-form promoter elements identified in the JGI 4.2 genome assembly, the associated promoter motif sequence for each hit is shown alongside

\begin{tabular}{|c|c|c|}
\hline Gene ID & Gene name & Motif \\
\hline ENSXETG00000003537 & plexd3 & GGGCGG \\
\hline ENSXETG00000008410 & c5orf4 & GGGCGG \\
\hline ENSXETG00000030719 & unknown1 & GGGCGG \\
\hline ENSXETG00000006282 & unknown2 & GGGCGG \\
\hline ENSXETG00000003943 & Irsam1 & CCGCCC \\
\hline ENSXETG00000027883 & c3orf10 & CCAAT \\
\hline ENSXETG00000028111 & unknown3 & CCAAT \\
\hline ENSXETG00000016171 & gata2 & CCGCCC \\
\hline ENSXETG00000029861 & unknown4 & CCAAT \\
\hline ENSXETG00000009337 & gdi3 & CCAAT \\
\hline ENSXETG00000012462 & gtf2e1.2 & CCAAT \\
\hline ENSXETG00000017744 & XB-GENE-5853280 & CCAAT \\
\hline ENSXETG00000004674 & eef1d & CCAAT \\
\hline ENSXETG00000004472 & mcts1 & CCAAT \\
\hline ENSXETG00000032447 & LOC100488751 & CCAAT \\
\hline ENSXETG00000000668 & $\mathrm{xkr5}$ & CCGCCC \\
\hline ENSXETG00000033055 & unknown5 & CCAAT \\
\hline ENSXETG00000007609 & thrsp & CCAAT \\
\hline ENSXETG00000002252 & unknown6 & CCAAT \\
\hline ENSXETG00000026459 & ywhaz & TATCA \\
\hline ENSXETG00000029162 & unknown7 & TATCA \\
\hline ENSXETG00000015053 & gdpd5 & TATCA \\
\hline ENSXETG00000009868 & tars & TATCA \\
\hline ENSXETG00000010686 & sepn1 & TATCA \\
\hline ENSXETG00000016524 & LOC100493317 & TATCT \\
\hline ENSXETG00000018194 & fam176a & TATCT \\
\hline ENSXETG00000009404 & adipor2 & CCAAT \\
\hline ENSXETG00000018026 & $\sec 22 a$ & AGATA \\
\hline ENSXETG00000002371 & kif27 & AGATA \\
\hline ENSXETG00000010991 & ercc4 & TATCT \\
\hline ENSXETG00000025304 & unknown8 & ATTGG \\
\hline ENSXETG00000002603 & gas2 & TATCT \\
\hline ENSXETG00000023254 & zfp3612.2 & TATCA \\
\hline ENSXETG00000009124 & clen7 & CCAAT \\
\hline ENSXETG00000018965 & crat.1 & CCAAT \\
\hline ENSXETG00000027013 & NP_001016033.1 & CCAAT \\
\hline ENSXETG00000027419 & a4galt & TATCA \\
\hline ENSXETG00000020165 & mkrn2 & CCAAT \\
\hline ENSXETG00000029144 & unknown9 & ATTGG \\
\hline ENSXETG00000030437 & tnrc6a & ATTGG \\
\hline ENSXETG00000018553 & XB-GENE-5960869 & TATCA \\
\hline
\end{tabular}

Table 3 Gene IDs and names of the immediately downstream genes of the $\mathbf{8 6}$ putative A-form promoter elements identified in the JGI 4.2 genome assembly, the associated promoter motif sequence for each hit is shown alongside (Continued)

ENSXETG00000016062

ENSXETG00000016933

ENSXETG00000014657

ENSXETG00000003950

ENSXETG00000030164

ENSXETG00000030426

ENSXETG00000022553

ENSXETG00000007987

ENSXETG00000023393

ENSXETG00000017669

ENSXETG00000013130

ENSXETG00000023739

ENSXETG00000007387

ENSXETG00000016524

ENSXETG00000013350

ENSXETG00000009412

ENSXETG00000000267

ENSXETG00000010533

ENSXETG00000007609

ENSXETG00000027421

ENSXETG00000014657

ENSXETG00000014963

ENSXETG00000019650

ENSXETG00000018194

ENSXETG00000029977

ENSXETG00000008526

ENSXETG00000033908

ENSXETG00000032885

ENSXETG00000019263

ENSXETG00000008969

ENSXETG00000022325

ENSXETG00000020057

ENSXETG00000007609

ENSXETG00000013463

ENSXETG00000015404

ENSXETG00000027433

ENSXETG00000021081

ENSXETG00000006922

ENSXETG00000033607

ENSXETG00000023477

ENSXETG00000003248

ENSXETG00000033920

\begin{tabular}{|c|c|}
\hline znf184 & GGGCGG \\
\hline ehmt1 & ATTGG \\
\hline slc25a30 & AGATA \\
\hline traf2 & CCGCCC \\
\hline NP_001120021.1 & AGATA \\
\hline unknown10 & TATCA \\
\hline fam120a & ATTGG \\
\hline $\arg 2$ & AGATA \\
\hline osbpl11 & TGATA \\
\hline usp21 & AGATA \\
\hline magi1 & TATCT \\
\hline wrb & CCAAT \\
\hline bmi1 & AGATA \\
\hline LOC100493317 & ATTGG \\
\hline $\operatorname{tfg}$ & ATTGG \\
\hline unknown11 & TATCT \\
\hline ccndx & CCAAT \\
\hline piwil2 & ATTGG \\
\hline thrsp & TGATA \\
\hline HIST1H4G & TGATA \\
\hline slc25a30 & ATTGG \\
\hline ctdsp1 & TGATA \\
\hline myh11 & AGATA \\
\hline fam176a & TATCT \\
\hline LOC100495404 & ATTGG \\
\hline LOC100495179 & GGGCGG \\
\hline UBE2U & AGATA \\
\hline P5F13_XENTR & ATTGG \\
\hline pdss2 & CCAAT \\
\hline $\operatorname{rad} 51 / 3$ & TATCA \\
\hline unknown12 & TATCA \\
\hline utp6 & CCAAT \\
\hline thrsp & TATCT \\
\hline zmynd12 & ATTGG \\
\hline shc1 & TATCT \\
\hline otop2 & ATTGG \\
\hline sgcg & GGGCGG \\
\hline ss18 & TATCA \\
\hline asxl1 & CCAAT \\
\hline hdhd3 & ATTGG \\
\hline strada & TGATA \\
\hline F166B_XENTR & CCGCCC \\
\hline
\end{tabular}


Table 3 Gene IDs and names of the immediately downstream genes of the $\mathbf{8 6}$ putative A-form promoter elements identified in the JGI 4.2 genome assembly, the associated promoter motif sequence for each hit is shown alongside (Continued)

\begin{tabular}{lll}
\hline ENSXETG00000010684 & dnajc19 & TGATA \\
ENSXETG00000027998 & prss8 & CCGCCC \\
ENSXETG00000010250 & chrnb3 & TGATA \\
\hline
\end{tabular}

Those selected for analysis are marked in bold.

we selected the gdi3 putative promoter, which contains a direct (i.e. present on the same strand as the downstream gene coding strand) CCAAT motif, for further characterisation and to test if it was also a target of the ilf3 containing transcription factor complex CBTF.

Upon co-incubation of an antibody raised against ilf3 the gdi3 complex was supershifted in EMSA, confirming the presence of ilf3 in the nucleic acid-protein complex (Figure 4a). The role of the gdi3 putative promoter element was also tested in vivo. To this end the expression profile of gdi3 was analysed using RT-PCR. Expression of gdi3 mRNA is absent until stage 11, then is expressed between stage 12 and 18, the latter of which it is at maximal, and from which its expression levels decrease until the last point sampled at stage 26 (Figure 4b). This expression wave occurs just after the maximal expression of gata2, a gene that is also controlled by the ilf3 transcription factor. A dominant-negative form of ilf3 (ilf3en) uses the fusion of ilf3 to the engrailed domain from Drosophila to repress transcription from any ilf3 binding site by recruitment of histone deacetylases [16]. This fusion has been shown to down-regulate gata2 mRNA levels when exogenouly expressed in Xenopus tropocalis embryos [8]. Synthetic mRNA encoding ilf3en was micro-injected into one-cell stage embryos before harvesting at stage 18 and total RNA was extracted, RTPCR was again used to analyse levels of gdi3 mRNA. Expression of gdi3 was ablated relative to levels of engrailed alone injected controls (Figure 4c), indicating ilf3 is involved in regulation of gdi3 in vivo at a transcriptional level.

\section{Conclusion}

We have previously identified and characterised a promoter element that requires an unusual A-form DNA structure in conjunction with a known promoter sequence motif. This combination of direct and indirect read-out mechanism drives early embryonic expression of the gata 2 gene in Xenopus and is responsive to the ilf3 containing transcription factor complex CBTF. However, the question of the prevalence of this type of regulatory mechanism in genomes remained. To address this we implemented a Perl program to investigate the occurence and used this to search the 4.2 version of the Xenopus genome. From the 86 hits obtained we selected five to test for both actual A-form structure and as specific targets for DNA binding proteins. All five of the selected targets were experimentally validated as A-form and as protein binding sites. One of these five, containing a

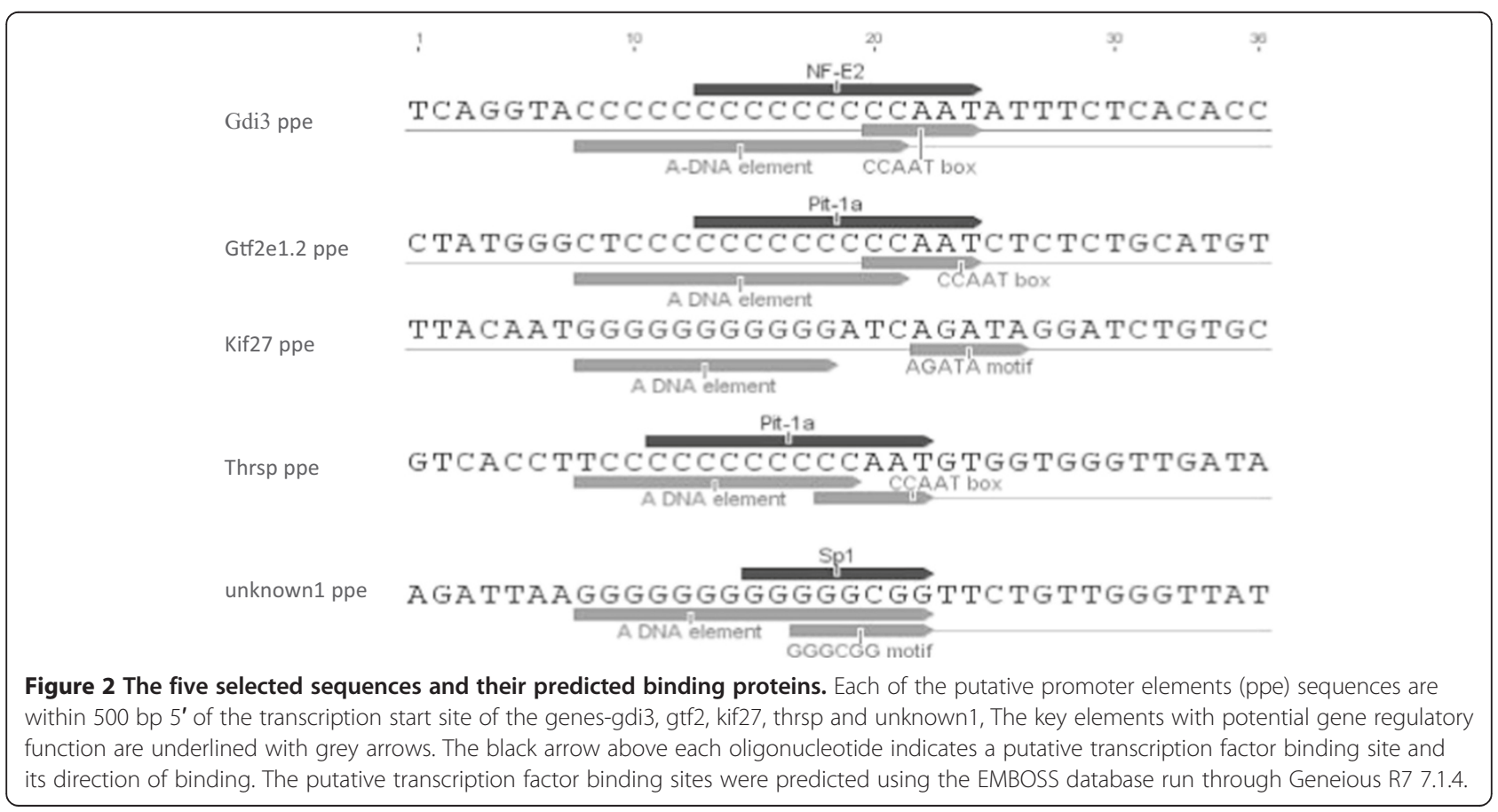


(a)

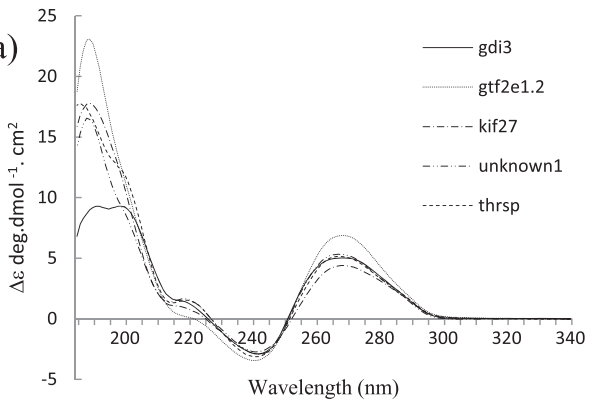

(c)

$5 `$ GTG CAT GCA TGC CCA ATG TCC ATC TCA ATG GGG GTT 3`

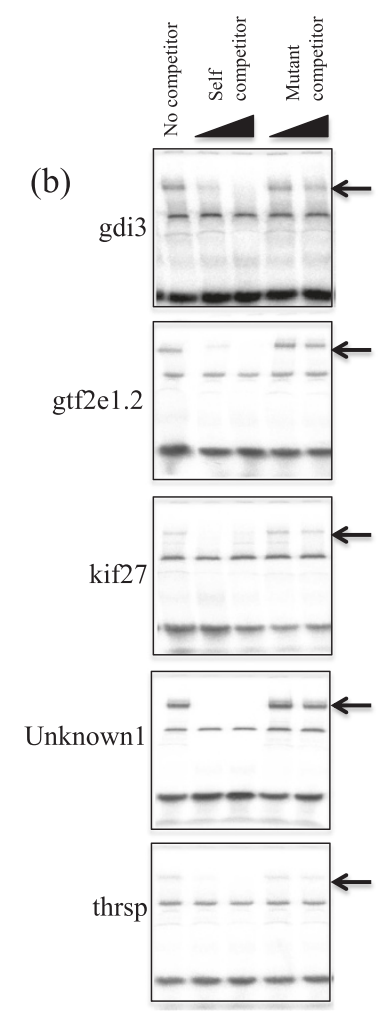

Figure 3 The putative promoter element is A-form and binds ilf3 in vitro. (a) Duplex 36 bp oligonucleotides corresponding to the five identified putative promoter elements display A-form DNA characteristics as observed by circular dichroism. (b) These duplex oligonucletides are shifted in EMSA experiments, these complexes are competed by titration of unlabelled self-competitor but not by CCAAT box containing B-form duplexes. The specific complexes are indicated by arrows. (c) The sequence of the B-form competitor used in the EMSA is shown, the CCAAT box is indicated in bold.

CCAAT motif as does the previously identified gata2 promoter, was selected for further validation. This element is the putative promoter for the gdi3 gene and was shown by supershift to be a target for the known gata 2 transcription factor ilf3. The temporal expression pattern of gdi3 occurs shortly after that of gata 2 and gdi3 transcription is also responsive to ilf3 fusion proteins in vivo. Taken together this is strong evidence for the element identified by the program to be a critical component of the promoter of gdi3.

Identification of the promoter elements required the A-forming potential of a base triplet of a given sequence to be calculated in a moving window along the genome using the method of Basham et. al. In the overwhelming majority of hits the APS consists of a consecutive sequence of Cs or Gs, with the first or second position in a block of Cs occasionally replaced by a $\mathrm{T}$. Only five cases were observed where this pattern does not hold, all involving repeated blocks of ATGC. However, it should be noted that APE values do not exist for 14 of the 64 possible triplets, which are effectively ignored by the present algorithm. The reliability of the method would no doubt be increased if these non-determined values were assigned. Despite this, apte provides a powerful tool for potential identification of A-form regulatory elements in whole genomes. A major problem in eukaryotic transcriptional studies is that transcription factor binding sites occur with high frequency and this leads to many 'false positive' identification of promoter elements by search programs. Potentially by considering DNA structure the reliability of such search programs could be significantly enhanced. For instance there are 25,253 CCAAT sequences (counting multiples per gene) within $500 \mathrm{bp}$ of a TSS in the 4.2 genome and 54,703 APS sequences anywhere in the genome. However there are only 36 in conjunction, a far more manageable number to screen.

Previous work on indirect read out mechanisms invoved with DNA recognition has largely been limited to in vitro experiments. Our validation of gdi3 as being regulated by such a mechanism is at least partially in vivo. Within eukaryotic genomes DNA is chromatinised with the interactions of the histones and the DNA, providing not only packaging but regulatory functions. It is unclear how non B-form DNA structures affects chromatinisation, possibly they chromatinise less well and 
(a)

$$
\begin{aligned}
& \text { Probe } \quad+++++ \\
& \text { Extract } \quad-+++ \\
& \text { Antibody } \quad-\quad+-\cdot \\
& \text { Wt competitor - - - + - } \\
& \text { Mt competitor - - - + }
\end{aligned}
$$

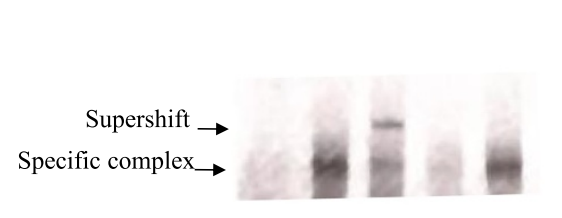

(b)

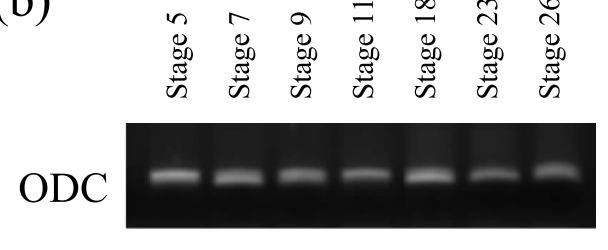

gdi3

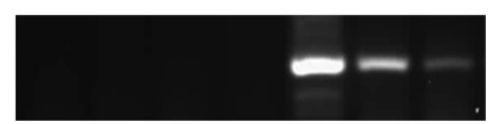

(c)

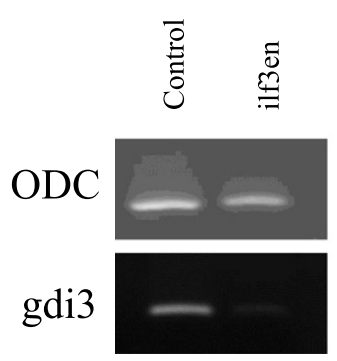

Figure 4 The expression of $g$ di3 mRNA is maximal at neurula stage and can be modulated by ilf3. (a) The complex gdi3 specific complex can be supershifted by addition of anti-ilf3 antibody. (b) The gdi3 gene expression is zygotic and peaks at neurula stage 18 when ilf3 is known to be nuclear and active. (c) Expression of gdi3 is ablated relative to an internal control, ODC, by exogenous expression a dominant-negative form of ilf3 (ilf3en) which acts at the transcriptional level.

are therefore bare regions at promoters, but the fact that we have identified a gene that is regulated in vivo by an A-form binding protein suggests that these structures persist within the chromatin environmment.

Although our results reflect mainly the identification of genes responsive to the ilf3 transcription factor potentially other A-form DNA binding proteins may also be recognising these elements. Importantly, the ability to look at whole genome assemblies means that it is now possible to study the role of these A-form elements within gene regulatory networks.

\section{Methods}

\section{Algorithm and implementation}

The algorithm is implemented as a Perl program named apte (A-form promoter transcription elements), which provides both a command-line interface and a Perl/Tk graphical interface. The program reads genomic sequence data from General Feature Format (GFF) Version 3 files (http://www.sequenceontology.org/gff3.shtml) and from Ensembl MySQL databases (http://www.ensembl.org/info/ data/ftp/index.html). GFF input files should contain a list of genes to be searched and the DNA sequence in FASTA format. Access to Ensembl databases is provided through the Ensembl Perl API (http://www.ensembl.org/info/docs/ api/index.html) which is a prerequisite for the program.
The main input parameters for apte are: motif, the promoter motif sequence; apelen, the minimum number of negative APE values in the APS; motifgap, the maximum number of bases between the APS and the motif; and genegap, the size of the region preceding the TSS to be searched. The default values adopted for the parameters are motif $=$ CCAAT, apelen $=10$, motifgap $=20$ and genegap $=500$. Searches can cover an entire genome or be limited to a specific gene or sequence region. Searches can also be made solely for A-DNA promoter sequences or promoter motifs. Results are output as a tab-separated table with a row for each combined sequence found, listing the APS and motif positions and summary details of the corresponding gene. Options are provided to write the results in GFF format; or in BED or WIG format files which may be uploaded to the Ensembl genome browser for display as custom tracks. The BED files indicate the location of the APS, the motif and the sign of the APE values over the search region. The WIG files plot the APE scores over the search region.

\section{Microinjection and RT-PCR}

Xenopus embryos were collected at time points during early developmental stages according to Nieuwkoop [17] and RNA extracted for RT-PCR analysis using the method of Steinbach and Rupp [18]. The samples were amplified to the linear phase of the amplification with 
the ODC gene used as an internal control, all primer sequences are available in supplemental information. Synthetic mRNA was prepared as previously described [8] and injected into both cells of two-cell stage embryos.

\section{Circular dichroism}

An Applied Photophysics $\mathrm{Pi}^{*} 180$ instrument was flushed with nitrogen gas (Oxygen-Free) for all $\mathrm{CD}$ experiments. Cell pathlengths of $1 \mathrm{~mm}$ and $4 \mathrm{~mm}$ were used to obtain far and near ultra-violet data respectively. Each duplex was dissolved in $100 \mathrm{mM}$ KF $5 \mathrm{mM} \mathrm{NaPO} 4$ buffer pH 7.6 at room temperature and stored on ice. Concentrations were determined by UV measurements at $260 \mathrm{~nm}$ coupled with snake-venom phosphodiesterase time course digestions to correct for hypochromic difference. The samples were run at $20+/-0.1 \mathrm{C}$ using a Melcor Peltier Thermoelectric Temperature Control Unit. Data was collected every $1 \mathrm{~nm}$ over the wavelength range $183 \mathrm{~nm}$ to $360 \mathrm{~nm}$ using adaptive sampling in conjunction with signal averaging in all cases. The instrument wavelength accuracy was $0.1+/-\mathrm{nm}$ determined from the Xeon lines and the ellipticity was calibrated from camphor suphonic acid at $290.5 \mathrm{~nm}$.

\section{Electrophoretic mobility shift assay (EMSA)}

DNA oligonucleotides (Invitrogen) were annealed to form duplexes and end-labeled by $\mathrm{T} 4$ polynucleotide kinase (NEB) using $\gamma^{33} \mathrm{P}$ ATP. The proteins were incubated with the nucleic acid probe for 15 minutes on ice in EMSA buffer [19] in the presence of $500 \mathrm{ng}$ poly dI-dC. Either wildtype or mutant non-labeled competitor was added at a 50 times excess to two of the reactions while a third reaction was incubated with anti-ilf3 antibody to allow identification of the specific DNA-protein complex. After incubation the DNA and DNA-protein complexes were separated on a $4 \%$ native polyacrylamide gel in $0.25 \mathrm{X}$ TBE. The gels were dried and visualized using a phosphorimager (Fuji).

\section{Competing interests}

The authors declare that they have no competing interests.

\section{Authors' contributions}

GS and MG conceived and designed the study. RT-PCR was performed by VR and LN conducted the EMSA assay while PC undertook the spectroscopic analysis. DW wrote and executed the APTE script. The manuscript was written jointly by DW and GS. All authors read and approved the final manuscript.

\section{Acknowledgements}

We would like to thank Dr Colin Sharpe for discussion concerning experimental procedure, and Mr Benjamin Marconnet (IUT Belfort) for contributions to the apte program. This work was supported by the Institute of Biomedical and Biomolecular Science, University of Portsmouth.

\section{Author details}

'Biophysics Laboratories, School of Biological Sciences, Institute of Biomedical and Biomolecular Science, University of Portsmouth, King Henry Building, King Henry I Street, Portsmouth PO1 2DY, UK. ${ }^{2}$ Centre for Molecular Design, School of Pharmacy and Biomedical Sciences, Institute of Biomedical and Biomolecular Science, University of Portsmouth, St Michael's Building, White Swan Road, Portsmouth PO1 2DT, UK.
Received: 5 March 2014 Accepted: 20 August 2014

Published: 26 August 2014

\section{References}

1. Mauro SA, Pawlowski D, Koudelka GB: The role of the minor groove substituents in indirect readout of DNA sequence by 434 repressor. J Biol Chem 2003, 278:12955-12960.

2. Chen $\mathrm{S}$, Gunasekera A, Zhang X, Kunkel TA, Ebright RH, Berman HM: Indirect readout of DNA sequence at the primary-kink site in the CAPDNA complex: alteration of DNA binding specificity through alteration of DNA kinking. J Mol Biol 2001, 314:75-82.

3. McGeehan JE, Streeter SD, Thresh SJ, Ball N, Ravelli RB, Kneale GG: Structural analysis of the genetic switch that regulates the expression of restriction-modification genes. Nucleic Acids Res 2008, 36:4778-4787.

4. Fairall L, Martin S, Rhodes D: The DNA binding site of the Xenopus transcription factor IIIA has a non-B-form structure. EMBO J 1989, 8:1809-1817.

5. Borden KL: The activating transcription factor region within the E2A promoter exists in a novel conformation. Biochemistry 1993, 32:6506-6514.

6. Llewellyn KJ, Cary PD, McClellan JA, Guille MJ, Scarlett GP: A-form DNA structure is a determinant of transcript levels from the Xenopus gata2 promoter in embryos. Biochim Biophys Acta 2009, 1789:675-680.

7. Parker SC, Hansen L, Abaan HO, Tullius TD, Margulies EH: Local DNA topography correlates with functional noncoding regions of the human genome. Science 2009, 324:389-392.

8. Scarlett GP, Elgar SJ, Cary PD, Noble AM, Orford RL, Kneale GG, Guille MJ: Intact RNA-binding domains are necessary for structure-specific DNA binding and transcription control by CBTF122 during Xenopus development. J Biol Chem 2004, 279:52447-52455.

9. Champ PC, Maurice S, Vargason JM, Camp T, Ho PS: Distributions of Z-DNA and nuclear factor I in human chromosome 22: a model for coupled transcriptional regulation. Nucleic Acids Res 2004, 32:6501-6510.

10. Brooks TA, Kendrick S, Hurley L: Making sense of G-quadruplex and i-motif functions in oncogene promoters. FEBS / 2010, 277:3459-3469.

11. Basham B, Schroth GP, Ho PS: An A-DNA triplet code: thermodynamic rules for predicting A- and B-DNA. Proc Natl Acad Sci U S A 1995 92:6464-6468.

12. Cazanove O, Batut J, Scarlett G, Mumford K, Elgar S, Thresh S, Neant I, Moreau M, Guille M: Methylation of Xilf3 by Xprmt1b alters its DNA, but not RNA, binding activity. Biochemistry 2008, 47:8350-8357.

13. Bass BL, Hurst SR, Singer JD: Binding-properties of newly identified Xenopus proteins containing dsRNA-binding motifs. Curr Biol 1994, 4:301-314

14. Bycroft M, Grunert S, Murzin AG, Procter M, St Johnston D: NMR solution structure of a double stranded RNA-binding domain from Drosophila staufen protein revels homology to the $\mathrm{N}$-terminal domain of ribosomal protein S5. EMBO J 1995, 14:4385-4391

15. Ramos A, Grünert S, Adams J, Micklem DR, Proctor MR, Freund S, Bycroft M, St Johnston D, Varani G: RNA recognition by a Staufen double-stranded RNA-binding domain. EMBO J 2000, 19:997-1009.

16. Ohkuma Y, Horikoshi M, Roeder RG, Desplan C: Binding site dependent direct activation and repression of in vitro transcription by Drosophila homeodomain proteins. Cell 1990, 61:475-484.

17. Nieuwkoop PD, Faber J: Normal Table of Xenopus laevis (Daudin). Amsterdam: North Holland Publishing Co; 1967.

18. Rupp R, Steinbach O: Quantitative Analysis of mRNA Levels in Xenopus Embryos by Reverse Transcriptase - Polymerase Chain Reaction (RT-PCR). In Molecular Methods in Developmental Biology: Xenopus and Zebrafish, Vol. 127. Edited by Guille M. New Jersey: Humana Press; 1998:41-56.

19. Orford R, Guille M: Bandshift Analysis using Crude Oocyte and Embryo Extracts from Xenopus Laevis. In Molecular Methods in Developmental Biology: Xenopus and Zebrafish, Vol. 127. Edited by Guille M. New Jersey: Humana Press; 1999:175-187.

doi:10.1186/1471-2105-15-288

Cite this article as: Whitley et al:: APTE: identification of indirect read-out A-DNA promoter elements in genomes. BMC Bioinformatics 2014 15:288. 